\title{
Imágenes del átomo: un producto del esfuerzo científico por comprender la estructura de la materia
}

The atom's images: a product of the scientific effort to understand the matter structure

$$
\text { P. Cabrera', A. Quiroga² y C. Naizaque }{ }^{3}
$$

Universidad Pedagógica Nacional. Facultad de Ciencia y Tecnología, Departamento de física. Ĺnea de investigación: la enseñanza de la física y la relación física matemática.

1paulita280@hotmail.com, 2'quimer 06@hotmail.com, ${ }^{3}$ nazapster@gmail.com

\section{Resumen}

En este escrito mostramos algunas de las representaciones del constituyente básico de la materia, siendo todas creaciones del intelecto humano, y no por ello son cuentos de hadas, por el contrario se encuentran basados en valores numéricos y datos experimentales, avalados por teorías aceptadas, no obstante al estar ubicadas en diferentes momentos históricos, suplían solo las necesidades de la época. Así pues, haciendo una revisión del concepto, se abarca la idea de átomo desde las ultimas concepciones de la mecánica dásica, su transición en lo que se conoce como una mezda entre la mecánica dásica y cuántica (mecánica cuántica de antaño), y la nueva representación que implico la visión de la mecánica cuántica, en adición a esto y haciendo alusión al tútulo, mostraremos un nuevo constituyente básico de la materia desarrollado a través de la manipulación del hombre denominados puntos cuánticos

\section{Palabras clave}

Átomo, modelo atómico de Rutherford, Thompson, Bohr, Sommerfeld, Schrödinger, Feynman, punto cuántico o átomo artificial.

\section{Abstract}

In this document we shown some of the representations of the matter's basic constituent, being all creations of the human intellect, but not for this are fairy stories, instead they are based in numerical value and experimental dates, endorsed by accepted theories, nevertheless to be placed in several historical moments, they supply only the time necessities. So, making a review of the concept, the atom idea is covered from the latest conceptions of classical mechanic, its transition between the dassic mechanic and the quantum (early quantum mechanics), and the new representation that involved the quantum mechanics vision, besides and making reference to the title, we will show a new matter basic constitute, developed through men's manipulation called quantum Dots.

\section{Keywords}

Atom, Rutherford's atomic model, Thompson, Bohr, Sommerfeld, Schrödinger, Feynman, quantum dot or artificial atom.

\section{Introducción}

Los átomos de la física moderna se parecen muy poco a los átomos de los antiguos, no son indivisibles ni macizos, como pensaba Demócrito, al contrario, la mayor parte del interior de un átomo está vaća. Así mismo los prinaipios básicos de la mecánica clásica no son aplicables a los de 
la cuántica, donde se tenían trayectoria definida por las cuales el electrón transitaba, tenemos ahora probabilidades de encontrar al electrón en determinadas regiones del espacio, o cuando hablábamos de cuerpos macizos y puntuales, descritos como tal, tenemos ahora descripciones de tipo ondulatorio, podemos describir sus comportamientos como de Broglie lo propuso en 1924, viendo al átomo ya no como un conjunto de cargas puntuales, descriptibles por la mecánica y la electrodinámica dásica, sino como un sistema vibrante ayyos comportamiento es descrito ahora por la mecánica y la electrodinámica cuántica. Rescatar en estos procesos de cambios conceptuales, nos parece pertinente para la enseñanza de la física. Ya que esta disciplina al igual que la física misma exige una revisión permanente de la forma como se presentan los conceptos que permiten describir los fenómenos físicos.

En este orden de ideas, la hipótesis atómica es quizá una de las concepciones físicas más divulgadas en el ámbito escolar, notamos que desde los primeros niveles de formación se incorpora la idea de átomo (Gentil, 1989; la Fuente, 2003; Rodríguez, 2007; De Cuéllar, 2008). Pese a esto, por lo general recurrimos a modelos atómicos pertenecientes a observaciones hechas hasta 1913 contempladas en el modelo de Bohr; haciendo a un lado la demás evidencia experimental que desde entonces se ha suscitado en el ámbito científico, lo que deja a la enseñanza de física actual lejos de su momento histónico.

En adición una reciente investigación que realizamos en algunas de las universidades de Bogotá nos muestra el poco trabajo que se ha realizado en este campo de estudio, esto, sumado a la carencia de modelos actuales, tratados aquí, generan de por si un problema en tomo a la investigación y por ende a la enseñanza de este tema (para ver detalladamente, Fig. 1. Esto radica en nuestra fuerte creencia de que las representaciones que quizás fueron y son eficientes para alguien (en nuestro caso nos referimos en particular al docente en física), pueden en gran medida contribuir a la construcción de modelos para los demás (por ejemplo para el alumno), cabe adara en este punto, que para lograr esta finalidad, es primordial la dara divulgación de la idea a transmitir entre otros factores.

a.) Trabajos de grado relacionados con átomos.

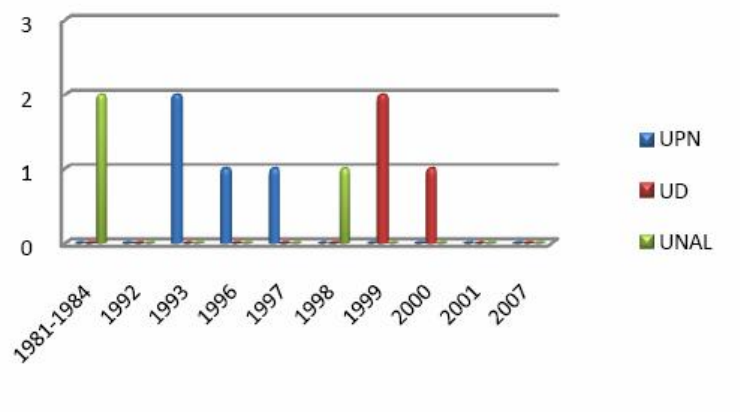

b.) Trabajos de grado relacionados con la nueva vision de átomo(QD).

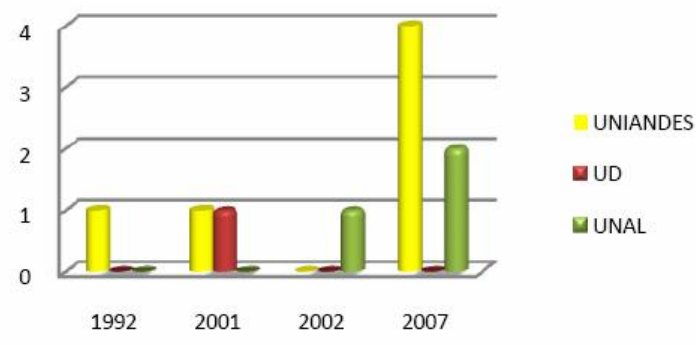

Figura No. 1 Resultados de la investigación hecha en las Universidades Pedagógica Nacional (UPN, que en el grafico se encuentra con color azul), Universidad Nacional de Colombia (UNAL, se muestra con color verde), Universidad Distrital Fráncico José de Caldas(UD, en el diagrama de barras se señala con color rojo), y Universidad de Los Andes(UNIANDES, en amarillo ). a.) Trabajos de grado relacionados con átomos, b.) Trabajos de grado relacionados con la nueva visión de átomo.

En este escrito mostramos algunas de las representaciones que han servido a los cient́ficos y a los individuos de la época, para tener una imagen del constituyente básico de la materia, su desarrollo, las dificultades que obligaron a construir uno nuevo, las evidencias experimentales, 
hasta llegar a las nuevas concepciones que se tienen de átomos, induidos los puntos cuánticos, o constituyente artificial de la materia, con el fin de presentar las imágenes que en la historia han surgido de átomo, enfatizando una de las más actuales que es la concepción del átomo como un sistema vibrante; resaltaremos además las investigaciones mencionadas anteriormente, mostrando la problemática en el ámbito universitario que creemos es una de las razones del por qué no se enseña en el ámbito escolar dichos modelos actuales.

\section{Representaciones del átomo}

Las evidencias experimentales y las teorías vigentes de cada época avalan diferentes modelos 0 interpretaciones las cuales aunque estén enmarcados en épocas pasadas, lejos de ser obsoletas, pueden describir diferentes fenómenos, como en su momento lo hicieron. Los aspectos novedosos de la física, necesitan de conceptos físicos igualmente novedosos 0 reformulaciones de aquellos elaborados por experiencias previas. Esto se ve reflejado en el proceso de conceptualización permanente de la idea de átomo; la cual, se ha revisado una y otra vez en la historia de la física como consecuencia del surgimiento de nuevas evidencias experimentales y teorías aceptadas. De modo que para evidenciar el proceso de reconceptualización de la idea de átomo mostramos una superficial recopilación de las imágenes científicamente aceptadas empezando desde J.J. Thompson hasta la concepción propuesta por Hisatsune Watanabe y Takeshi Inoshita (Nossa, 2007).

Imagen de J.J. Thompson

Propuso una descripción tentativa, en ella considera una distribución de cargas positivas y negativas, la primera de estas se suponía en forma esférica aryo radio era aproximadamente 10$10 \mathrm{~m}$ siendo este el tamaño del átomo y pequeños electrones incrustado "un pastel de pasas" (este es el nombre que se le da en la literatura), que describían pequeñas vibraciones cuando estos estaban excitados. Este modelo, era suficiente para la descripción de deflexión de las partículas alfa para ángulos pequeños, gracias a la acción de la fuerza de Coulomb y dejaba de serlo para ángulos grandes.

\section{Imagen de Rutherford}

Rutherford retoma esta problemática y construye un nuevo modelo, que tiene en cuenta la proporcionalidad inversa entre la fuerza de Coulomb y el radio, plateando así que la carga positiva estaría concentrada en una región auarto órdenes de magnitud menor a la que denomino núdeo. Esta pequeña corrección daba solución al problema, pero sus falencias se vieron representadas en la electrodinámica dásica ya que para esta, una carga que está siendo acelerada emitińa radiación electromagnética constantemente originando que el electrón decayese al núdeo en aproximadamente $10^{-12}$ segundos.

\section{Imagen de Bohr.}

En 1913, Bohr dio solución al problema de estabilidad planteado en el modelo de Rutherford, gracias a la predicción de los espectros de radiación. Proponiendo, que dicha radiación seria un producto de la transición (saltos) entre niveles de energía a los que llamo estacionarios, ya que cada uno de estos niveles muestra una energía permitida constante, y los saltos son entonces el producto de absorción o emisión del electrón en un átomo. Pero a pesar de describir las líneas espectros que Balmer había consignado en su formula empírica este modelo ya no era suficiente para explicar la estructura fina e hiperfina, presentes en los espectros. 
Imagen de Sommerfeld

Sommerfeld planteo un modelo basado en las orbitas Keplerianas, en donde en uno de sus focos se encontraba el núcleo, además ya no solo aparecía el numero cuántico principal n, sino que se introducían dos números auánticos subordinados nr (dependiente del radio) y nq (azimutal), la suma de estos dos daba cuenta de n, obteniendo así circunferencias (las mismas que predecía Bohr) y elipses (indicarían la estructura fina presente en el espectro), cabe adarar que los números aúnticos por separado no tenían un sentido, sin embargo su suma era lo realmente importante, obteniéndose de esta forma estados degenerados, y adicionalmente dándole sustento teórico a los desdoblamientos al induir parámetros relativistas. Con estos dos aspectos que se mencionaron se resuelven los problemas de la correctividad entre el experimento y la teoría (momentáneamente), develándose la estructura final (Sánchez, 2000).

\section{Imagen de Schrödinger}

En 1927 después de que Lande, y Pauli hicieran las pertinentes correcciones al modelo que Sommerfeld introduciendo el concepto de espín, Schrödinger postulo una imagen de átomo, ubicada en el mundo de las probabilidades, basada en los datos experimentales y la teoría de la época, donde fundamentado en el principio de incertidumbre de Heisemberg, elimina las trayectoria trabajadas en modelos anteriores, y se limita a una descripción probabilística para encontrar al electrón en determinadas regiones del espacio. Esta es la imagen adoptada en la actualidad y es conocido como el átomo mecánico.

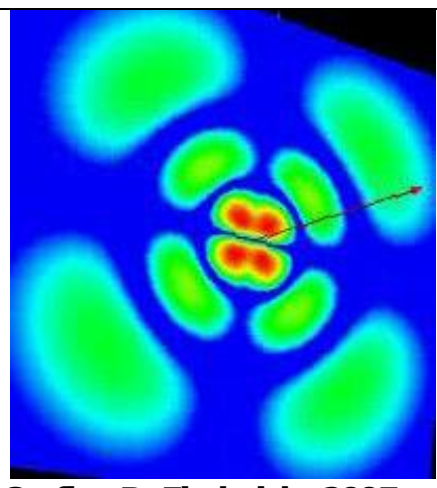

Grafico B. Fiederich, 2005

\section{Imagen de Feynman}

Cabe notar, que este modelo está enmarcado en la electrodinámica cuántica, y tiene la particularidad de es estar ubicado en un diagrama espacio-temporal, en el cual se ubica el protón y electrón, cada uno con su propia línea de mundo presentando además interacciones a partir de fotones virtuales portadores de fuerza electromagnética (Feynman, 1985).

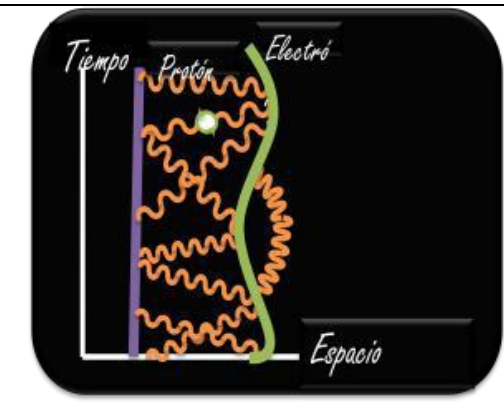

Grafico C. (Feynman, 1985)

\section{Imagen de Punto cuántico o átomo artificial}

Es un confinado de electrones no natural que actúa bajo la influencia de potenciales no colombianos, si no que el atrapamiento ocurre cuando se ponen diferencias de potencial laterales, paradójicamente esto átomos artificiales están hechos de átomos naturales, a pesar de lo anteriormente mencionado existen propiedades las auales han ayudado a fortalecer la analogía existente entre ellos y los átomos naturales algunas de estas son: Existencia de pequeñas regiones espaciales del orden de nanómetros que confinan o atrapan electrones en las tres dimensiones; los niveles de energía son discretos debido al confinamiento; Es posible confinar desde uno hasta cientos de electrones, y obtener diferentes formas, como las que se muestran en la grafica, además de poseer otras auantas. 


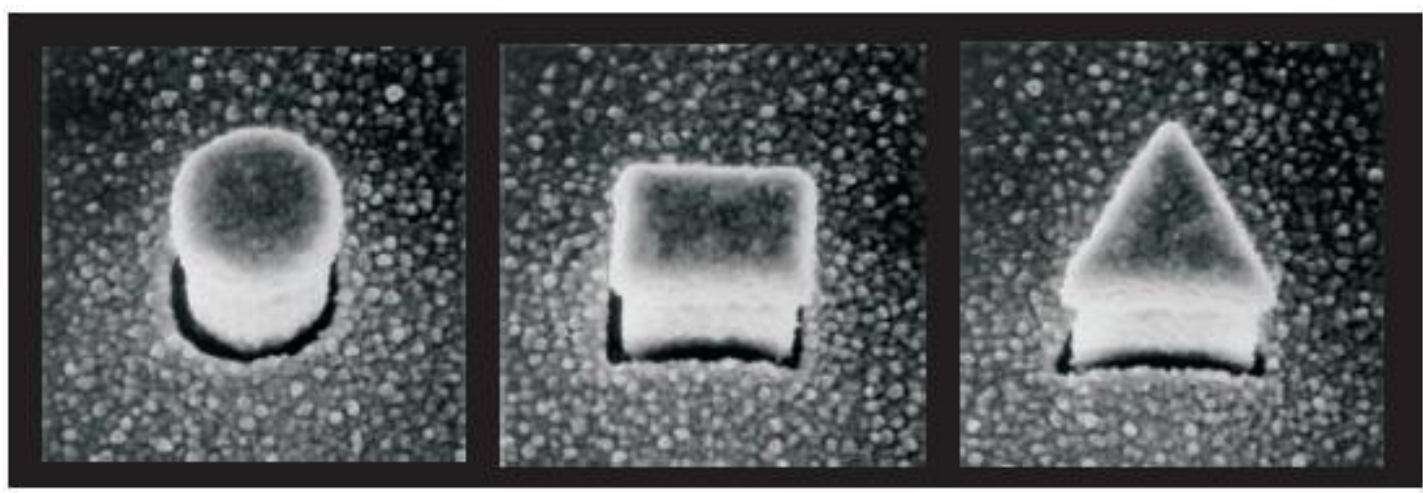

Estas son las representaciones que se tienen de cada modelo y que son mostradas de forma Ya sea un poco más formal o no, y a pesar de ser ciertas en su totalidad, dejan de lado muchos aspectos históricos, que conllevan a comprender mejor cada visión, aspectos tales como: los órdenes de magnitud para los cuales es posible aplicar cada modelo, que velocidades maneja cada modelo, que modelos atómicos se ajustan a las necesidades de cada área de estudio, tales como: la química, la tecología e incluso que modelo es suficiente para describir cada fenómeno físico como el ferromagnetismo, diamagnetismo, los fenómenos térmicos, entre otros. Nuestro trabajo compila todas estas características enfocándonos en las representaciones visuales o imágenes como un elemento central o elemento director en el análisis histórico del concepto de átomo.

\section{Conclusiones}

En la investigación a la que hacemos alusión se ve una problemática, esta muestra que a nivel local los trabajos de grado hechos en las universidades Distrital y Pedagógica, en las que se está formando licenciados en física, no existe un estudio ni una propuesta, metodológica o didáctica que abarque el concepto de átomo desde ideas dásicas hasta la actual de átomo. Lo que nos lleva a auestionamos, acerca de que es lo se lleva al aula, si nosotros como docentes no tenemos daridad acerca de temas tan básicos como estos, y nuestros conocimientos no se encuentran actualizados, ¿como hacer que el estudiante comprenda estos temas? 0 acaso es que la ćísica de hace un siglo es más pertinente en el proceso de enseñanza aprendizaje? La respuesta a esta preguntas han sido debatida en el artículo Why we should teach the Bohr model and how to teach it effectively(McKagan, 2008) sin embargo esta bien auestionamos, y pensar hasta qué punto es pertinente involucrar al estudiante en temas actuales de la física, si la complejidad de las nuevas teorías son un impedimento para que el estudiante aceda a este conocimiento, o si estamos subestimándolos y de paso quitando les la posibilidad de que se hagan participes de su momento histórico, añadiendo a esto último que labor docente está basada también en hacer mas inteligibles los procesos cient́ficos cabe questionamos si estamos haciendo bien nuestra labor.

Bibliografía

Cuéllar, F. (2008). El modelo atómico de E. Rutherford, del saber científico a conocimiento escolar. Enseñanza de las ciencias. 26(1), 43-52

De La Fuente, A. (2003). Estructura atómica: análisis y estudio de las ideas de los estudiantes (8 de EGB). Enser̃anza de las ciencias. 21(1), 123-134.

Feynman, R. (1985). Electrodinámica cuántica. Princeton: Ed. Alianza.

Fiederich H; Brooner, H y Roht. (2005). Imágenes del átomo de hidrógeno. CD- ROM. Alemania: Universidad Kartsruhe

Gentil, C. (1989). Nivel de apropiación de la idea de discontinuidad de la materia en alumnos de bachillerato. Implicaciones didácticas. Enseñanza de las ciencias. 7(2), 126- 131. 
Nossa, J. (2007). Meta-materiales basados en puntos cuánticos: geometría y acoplamiento: Bogotá: Universidad De Los Andes (Tesis de maestría). Rodríguez, Pozas, Sánchez y otros. (2007). Química. $2^{\circ}$ Bachillerato. MoGraw-Hill.

Sánchez, J. (2001). Historia de la mecánica. I. El periodo fundacional. (1860-1926). Barcelona: Crítica. 